\title{
An Adaptive Fuzzy Contrast Enhancement Algorithm with Details Preserving
}

\author{
Jing Rui Tang \& Nor Ashidi Mat Isa* \\ Imaging and Intelligent System Research Team (ISRT), \\ School of Electrical and Electronic Engineering, Engineering Campus, \\ Universiti Sains Malaysia, 14300 Nibong Tebal, Penang, Malaysia \\ Email: ashidi@usm.my
}

\begin{abstract}
This paper modifies the Adaptive Contrast Enhancement Algorithm with Details Preserving (ACEDP) technique by integrating a fuzzy element in the image type selection. The proposed technique, named the Adaptive Fuzzy Contrast Enhancement with Details Preserving (AFCEDP) technique, first computes the degree of membership of the input image to three categories, i.e. low-, middle- or high-level images. The AFCEDP technique then clips the histogram at different plateau limits that are computed from both the degree of membership and the clipping functions. The classification of an image in the ACEDP technique is done based solely on the intensity range of the maximum number of pixels, which may be inaccurate. In the proposed AFCEDP technique, the image type classification is handled in a better way with the integration of a fuzzy element. The performance of the proposed AFCEDP technique was compared with the conventional ACEDP technique and several state-of-art techniques described in the literature. The simulation results revealed that the AFCEDP technique demonstrates good capability in contrast enhancement and detail preservation. In addition, the experiments using cervical cell images and HEp-2 cell images showed great potential of the AFCEDP technique as a technique for enhancing medical microscopic images.
\end{abstract}

Keywords: contrast enhancement; detail preservation; grayscale image; histogram clipping; fuzzy membership

\section{Introduction}

Image contrast enhancement remains an important challenge in the field of image processing. It plays an important role in many fields, including robotics, astronomy, biomedical and military applications. Contrast enhancement yields an image with improved visual quality by changing the pixel intensities of the image.

There are various techniques available for contrast enhancement. Conventional histogram equalization ( $\mathrm{HE}$ ) is one of the most widely used techniques owing to its effectiveness and ease of implementation [1]. It remaps the gray levels of the

Received October $15^{\text {th }}, 2014$, Revised November $27^{\text {th }}, 2014$, Accepted for publication November $27^{\text {th }}, 2014$. Copyright (C) 2014 Published by ITB Journal Publisher, ISSN: 2337-5787, DOI: 10.5614/itbj.ict.res.appl.2014.8.2.4 
image based on a probability density function (PDF) to flatten and stretch the dynamic range of the histogram [2]. Despite its effectiveness in increasing the image contrast, HE generates unwanted artifacts and provides unnatural visual effects in the image since it always shifts the mean brightness of the image to the middle gray level, regardless of the input mean brightness [3]. In addition, it often causes saturation in the image and consequently leads to a loss of information [4].

Different approaches have been introduced to overcome the limitations of HE in the preservation of mean brightness and image details. In this paper, a modification of the conventional ACEDP [5] is introduced, which integrates a fuzzy element in the categorization of the image type. The fuzziness enables the entire pixel intensity distribution to be taken into consideration during the selection of the clipping limits. The belonging of an image to one of several image type categories is described in terms of its degree of membership, which helps to improve the conventional ACEDP in both detail preservation and contrast enhancement.

The rest of this paper is organized as follows: Section 2 briefly presents some related works. Section 3 describes the details of the proposed technique. Section 4 discusses the performance matrices used to evaluate the AFCEDP technique, and Section 5 presents simulation results and discussions for both standard images and medical images (i.e. cervical cell images and HEp-2 cell images). Finally, Section 6 concludes our work.

\section{$2 \quad$ Related Works}

Various techniques have been proposed to resolve the limitations of HE. The first solution to overcome the problem of the mean brightness shifting was proposed by Kim [6]. The proposed Brightness Preserving Bi-Histogram Equalization (BBHE) technique proved to successfully reduce the shifting of the mean brightness by dividing the input histogram into two sub-histograms at the mean brightness of the image. The resultant images have less saturation effect, unnatural enhancement and unwanted artifacts [7]. Following BBHE, numerous techniques have been proposed that work based on histogram segmentation. For example, there are techniques that employ bi-histogram equalization (e.g. Dualistic Sub-image Histogram Equalization (DSIHE) [2] and Minimum Mean Brightness Error Bi-histogram Equalization (MMBEBHE) [8]) and techniques that employ multi-histogram equalization (e.g. Recursive Mean-Separate Histogram Equalization (RMSHE) [9], Recursive Sub-image Histogram Equalization (RSIHE) [10], Dynamic Histogram Equalization (DHE) [4] and Brightness Preserving Dynamic Histogram Equalization (BPDHE) [11]). 
Apart from concentrating on the issue of mean brightness shifting, modification of HE has also been performed to retain the information in the image. Modified Histogram Equalization (MHE) alters the accumulations in the input histogram before histogram equalization is applied in order to enhance the contrast of the image, while preserving the discrete structures and fine details of the image [12]. Adaptive Histogram Equalization Algorithm (AHEA) uses information entropy as the target function and introduces a new parameter in the histogram equalization formula to adaptively adjust the spacing of two adjacent gray levels in the output histogram based on the type of input image [13].

Another type of histogram-equalization-based technique is Clipped Histogram Equalization (CHE). Histogram clipping is performed to control the enhancement rate and thus avoiding intensity saturation in the image. Bihistogram Equalization Plateau Limit (BHEPL) combines BBHE with the idea of clipped histogram equalization [7]. Other examples of histogram clippingbased equalization techniques include Bi-histogram Equalization Median Plateau Limit (BHEPL-D) [14], Brightness Preserving Plateau Limits Histogram Equalization (BPPLHE) [15] and Image Enhancement Using Exposure Based Sub Image Histogram Equalization (ESIHE) [16]. Recently, the Adaptive Contrast Enhancement Algorithm with Details Preserving (ACEDP) was proposed, where different histogram clipping functions are implemented based on image type [5]. The ACEDP technique first classifies the type of image based on the distribution of the maximum number of pixels according to their intensities followed by defining a function for each image type to set the plateau level. The image type classification and the corresponding plateau levels from the ACEDP technique are shown in Figure 1.

Motivated by the idea of histogram clipping and the convincing results from the literature, we propose a modification of the conventional ACEDP in order to improve its capability in detail preservation and contrast enhancement. The proposed AFCEDP technique is described in detail in the following section.

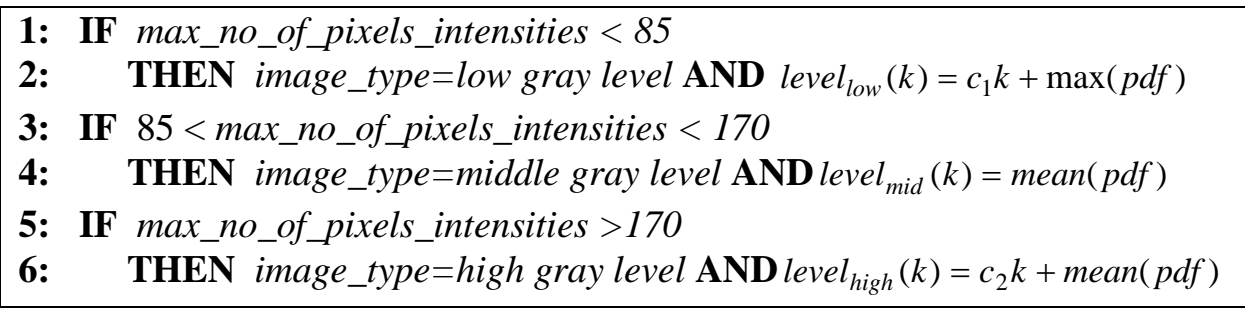

Figure 1 Classification and clipping functions in ACEDP [5]. 


\section{Proposed Technique}

The conventional ACEDP classifies the image type based solely on the maximum intensities of the pixels. In order to improve its ability in defining the image type as well as in choosing a suitable clipping limit, a modified version of the conventional ACEDP technique, named AFCEDP, is proposed, which integrates a element of fuzziness during the classification process. The proposed AFCEDP algorithm comprises of the following stages:

1. Determination of the membership function

2. Computation of the degree of membership

3. Defining three plateau functions and computation of the clipping limit

4. Clipping and equalization of the histogram

\subsection{Determination of the Membership Function}

The determination of the image type in the conventional ACEDP is based on the intensity range owned by the maximum number of pixels in the image. The proposed AFCEDP technique defines a trapezoidal-shaped membership function at threshold values of 85 and 170. Eqs. (1) to (3) are the membership functions for low-, middle- and high-level images respectively. Figure 2 illustrates the whole membership function.

$$
\begin{aligned}
& \mu_{\text {low }}(k)=\left\{\begin{array}{cl}
0 & , \text { for } k>95 \\
\frac{95-k}{20} & , \text { for } 75 \leq k \leq 95 \\
1 & , \text { for } k<75
\end{array}\right. \\
& \mu_{\text {mid }}(k)=\left\{\begin{array}{cl}
\frac{k-75}{20} & , \text { for }(k<75) \cup(k>180) \\
1 & , \text { for } 75 \leq k \leq 95 \\
\frac{180-k}{20} & , \text { for } 160 \leq k \leq 180
\end{array}\right. \\
& \mu_{\text {high }}(k)=\left\{\begin{array}{cl}
\frac{k-160}{20} & , \text { for } k<160 \\
\frac{k-160}{1} & , \text { for } k>180 \leq k \leq 180
\end{array}\right.
\end{aligned}
$$

where $k$ is the intensity of the pixels in the image. 


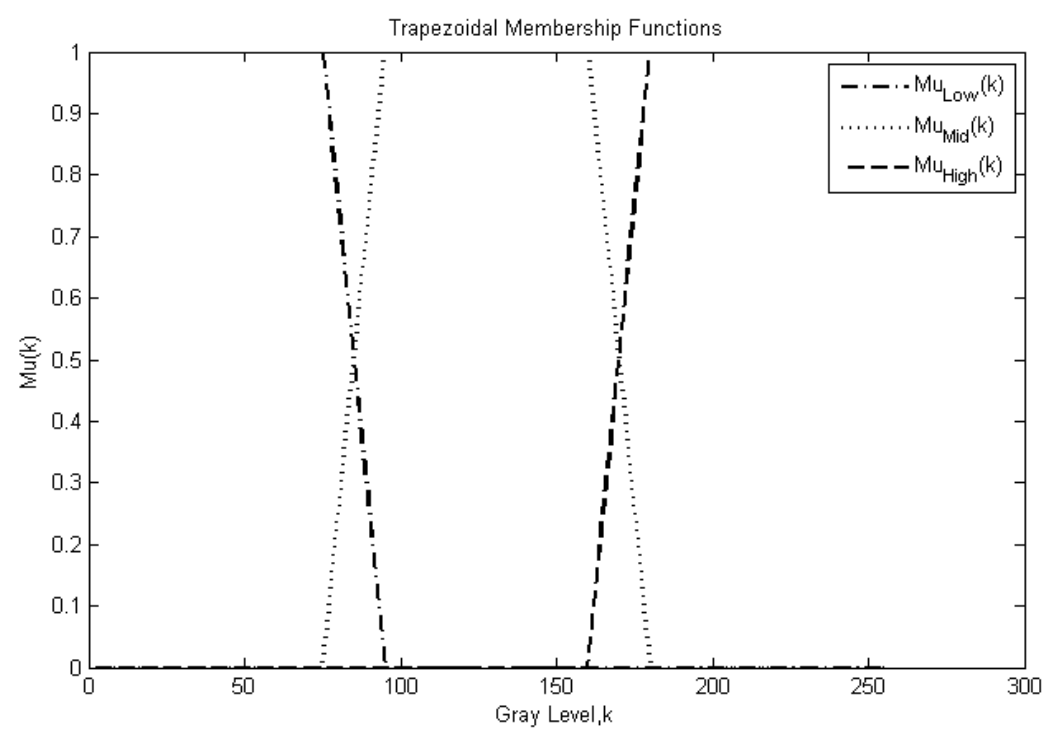

Figure 2 Trapezoidal membership functions.

\subsection{Computation of the Degree of Membership}

In order to obtain the reference intensity for the trapezoidal membership function, the degree of belonging of an image to the three categories using partition is computed. The three categories low-, middle- and high-level images, previously implemented in the conventional ACEDP, are used as reference. The reference intensity, $\lambda$, of the image can be computed using Eq. (4).

$$
\lambda=(\text { low_part } \times 43)+(\text { mid_part } \times 213)+(\text { high_part } \times 128)
$$

For instance, an image with 0.2 partitions of pixels with low-level intensities, 0.15 partitions of pixels with middle-level intensities and the remaining 0.65 partitions of pixels with high-level intensities, is a relatively bright image as more than half of its pixels have intensities greater than 170 gray levels. By using Eq. (4), the reference intensity, $\lambda$, of this image is 166.25.

\subsection{Defining Three Plateau Functions and Computation of the Clipping Limit}

The AFCEDP technique employs the same clipping functions as the conventional ACEDP technique. As described in the conventional ACEDP, the acceptable range for the slopes $c 1$ and $c 2$ is [-0.015,-0.005] and [0.005,0.007] respectively. In the proposed AFCEDP technique, the same values for $c 1$ and $c 2$ as employed in the conventional ACEDP technique are used for a fair 
comparison of the final results. The values for $c 1$ and $c 2$ are -0.01 and 0.007 respectively. Based on the reference intensity, $\lambda$, and the plateau functions in Figure 1, the AFCEDP technique implements the final clipping function, $\sigma(k)$, using Eq. (5).

$$
\sigma(k)=\left[\mu_{\text {low }}(\lambda) \times \text { level }_{\text {low }}(k)\right]+\left[\mu_{\text {mid }}(\lambda) \times \text { level }_{\text {mid }}(k)\right]+\left[\mu_{\text {high }}(\lambda) \times \operatorname{level}_{\text {high }}(k)\right]
$$

\subsection{Clipping and Equalization of the Histogram}

The clipping function $\sigma(k)$ provides the clipping limit at each gray level. Consider an input grayscale image. The histogram of the image, $H(k)$ is defined as:

$$
H(k)=n_{k} \text {, for } k=0,1, \ldots, L-1
$$

where $n_{k}$ is the occurrence of intensity $k$ in the image and $L$ is the total number of gray levels in the image. The probability density function (PDF) of the image, $p(k)$ is defined as:

$$
p(k)=\frac{H(k)}{N}, \text { for } k=0,1, \ldots, L-1
$$

where $N$ is the total number of pixels in the image.

The cumulative density function (CDF), $c(k)$ is defined as:

$$
c(k)=\sum_{i=0}^{k} p(i) \text {, for } k=0,1, \ldots, L-1
$$

HE involves mapping input gray level $k$ into output gray level $f(k)$, where the transformation function $f(k)$ is defined as:

$$
f(k)=X_{0}+\left(X_{L-1}-X_{0}\right) \cdot c(k)
$$

where $X_{0}$ and $X_{L-1}$ represent the lowest and highest gray levels respectively. HE remaps the input image into the entire dynamic range $\left[X_{0}, X_{L-1}\right]$.

In the proposed AFCEDP technique, the PDF of the input image is limited to the clipping function, as shown in Eq. (10).

$$
n e w_{-} p(k)=\min [p(k), \sigma(k)] \text {,for } k=0,1, \ldots, \lambda-1
$$

The CDF of the enhanced image can be computed using Eq. (11), 


$$
c(k)=\sum_{i=0}^{k} n e w_{-} p(i) \text {, for } k=0,1, \ldots, L-1
$$

Finally, the transformation function of the AFCEDP technique can be represented as Eq. (9), with the CDF computed from Eq. (11).

\section{Performance Evaluation}

Evaluation of the enhancement results was performed by qualitative and quantitative analysis. For performance comparison, seven histogramequalization-based techniques were implemented, namely conventional Histogram Equalization (HE), Bi-histogram Equalization Median Plateau Limit (BHEPL-D), Brightness Preserving Plateau Limit Histogram Equalization (BPPLHE), Modified Histogram Equalization (MHE), Image Enhancement Using Exposure Based Sub Image Histogram Equalization (ESIHE), Adaptive Histogram Equalization Algorithm (AHEA) and the conventional Adaptive Contrast Enhancement Algorithm with Details Preserving (ACEDP). All these techniques were tested with 90 benchmark images that were downloaded from a public image database.

\subsection{Qualitative Analysis}

The qualitative analysis evaluated the resultant images using the naked eye. The enhanced images were observed visually to investigate the capabilities of the different enhancement techniques. In this case qualitative analysis was performed by examining the amount of information details in the image, the degree of contrast enhancement as well as the homogeneity of regions in the image and the naturalness of the image.

\subsection{Quantitative Analysis}

The quantitative analysis, involving numerical evaluation, was performed to further support the qualitative analysis. Three objective functions, namely entropy, output-input standard deviation and contrast improvement evaluation were employed. The ability of the proposed techniques in retaining details in the image was measured using entropy. Evaluation of the output-input standard deviation and contrast improvement was employed in order to evaluate the contrast enhancement.

According to information theory, Shannon entropy can be used to measure the richness of information [17]. This approach is widely used to measure the amount of details contained in images [2, 14, 18]. The entropy, E, of an image can be calculated using Eq. (12), 


$$
E=-\sum_{i=1}^{N} r(i) \log _{2} r(i)
$$

A higher entropy value is desirable because it indicates that the technique is better at retaining image details. The entropy of an image can only be compared for the same image, i.e. before and after processing. Therefore, it is meaningless to compare the entropy values of two different images.

The output-input standard deviation, SD, which is a measurement for the difference between the standard deviation of the enhanced image and the standard deviation of the input image [19], can be computed using Eq. (13),

$$
S D=S D_{\text {Outputmage }}-S D_{\text {InputImage }}
$$

In addition to the output-input standard deviation, the deviation of gray levels in the image can be used as a contrast improvement investigator. This deviation is known as contrast improvement evaluation, C. It can be computed and subsequently converted into decibels $(\mathrm{dB})$ using the image contrast function as shown in Eq. (14) [20, 21],

$$
C=10 \log _{10}\left[\frac{1}{W H} \sum_{u=1}^{W} \sum_{v=1}^{H} g^{2}(u, v)-\left|\frac{1}{W H} \sum_{u=1}^{W} \sum_{v=1}^{H} g(u, v)\right|^{2}\right]
$$

where $W$ and $H$ are the width and height of the image respectively, $g(u, v)$ is the intensity of the pixel at a 2-dimensional position $(u, v)$.

Similar to entropy measurement, for both the output-input standard deviation and the contrast improvement evaluation, a larger value is desirable as it indicates a better contrast enhancement ability.

\section{$5 \quad$ Results and Discussions}

The simulation results of the proposed AFCEDP technique and a comparison with the other seven techniques are presented first. These are followed by the simulation results of the AFCEDP technique on medical microscopy images, i.e. cervical cell images and HEp-2 cell images, for further investigation of its robustness and feasibility.

\subsection{Standard Test Images}

Two randomly selected test images, namely Car and Fish, are presented in Figures 3 and 4 respectively for qualitative analysis. 
For the first test image, Car, the proposed AFCEDP technique produces an image that is comparable to that produced by the BHEPLD, BPPLHE and AHEA techniques, as shown in Figure 3(c), 3(d) and 3(g) respectively. The resultant image as depicted in Figure 3(i) looks natural with a minimal amount of over-enhancement. The resultant image produced by the MHE technique is similar to the image enhanced by the ACEDP technique. Both of these images appeared to be darker as compared to the input image. This shifting of mean brightness is undesired. For the image enhanced with the ESIHE technique, the contrast improvement is less significant compared to the image enhanced with the proposed technique. In terms of detail preservation, most information of the image enhanced with the proposed AFCEDP technique is well retained. This can be seen by observing the texture of the house as well as the details of the car. These are well retained, as highlighted at the center of the image. Moreover, the AFCEDP technique produces less non-homogenous regions. This unwanted effect can be observed in Figure 3(b). Both the top (i.e. skies) and the bottom (road) of the image enhanced by the HE technique demonstrate many small regions. Apart from producing less non-homogenous regions, the proposed AFCEP technique is better than the conventional ACEDP technique in reducing the non-homogeneity of the regions, as depicted in the dashed boxes.

The mean brightness of the image enhanced using the HE technique for the test image Fish is significantly changed when compared to the input image shown in Figure 4(a). However, this effect is less strong in the image enhanced using the proposed AFCEDP technique. Other images appear to be similar, particularly the images enhanced using the BHEPLD, BPPLHE, ESIHE and AHEA techniques. The mean brightness of these images looks the same as those in the proposed AFCEDP technique. However, the proposed AFCEDP technique yields better image enhancement than the conventional ACEDP technique, which can be seen through the homogeneity of the images. There are many unwanted small regions present in the background of the image enhanced when using the conventional ACEDP technique, as highlighted in Figure 4(h) in the dashed boxes in the image background. The proposed AFCEDP technique also produces an image that appears to be darker when compared with the conventional ACEDP and MHE techniques. This successfully prevents the saturation effect on the fish scales and thus reduces the occurrence of artifacts.

Observation of the two test images reveals that the proposed AFCEDP technique demonstrated a performance comparable to that of the other histogram-equalization-based techniques. It performed better than the conventional ACEDP technique in terms of detail preservation and contrast enhancement. 
The quantitative analysis of the 90 test images downloaded from the image database [22] was conducted by implementing all the techniques and computing the average values for the three objective evaluation functions. The average results of the quantitative analysis are tabulated in Table 1 . For each of the analysis outcomes, the best result is made bold.

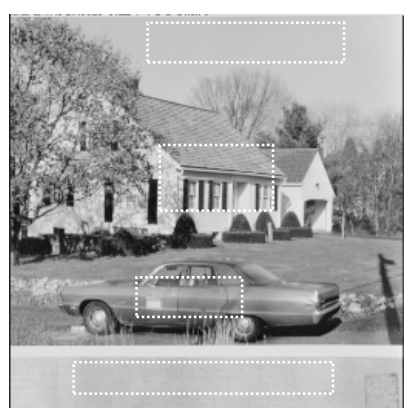

(a)

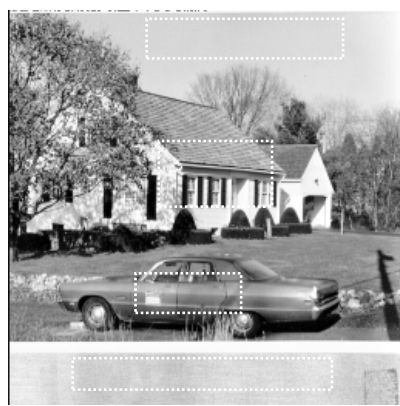

(d)

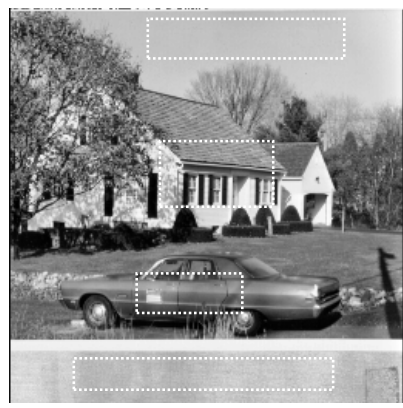

(g)

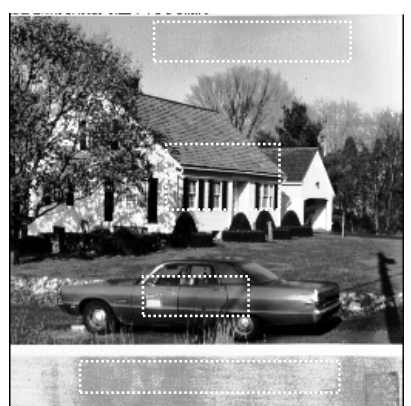

(b)

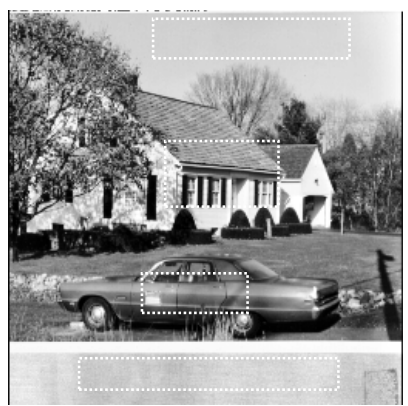

(e)

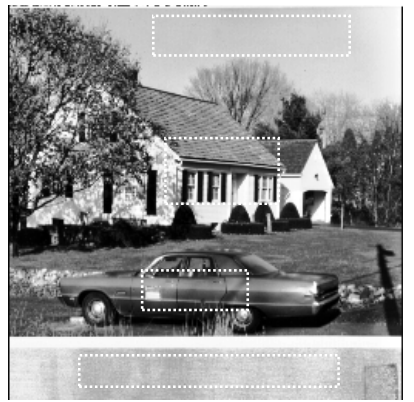

(h)

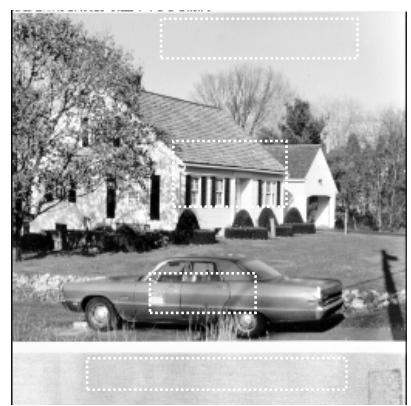

(c)

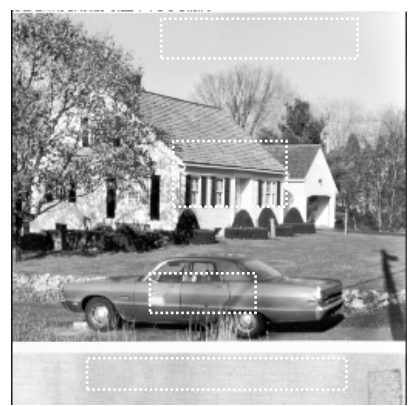

(f)

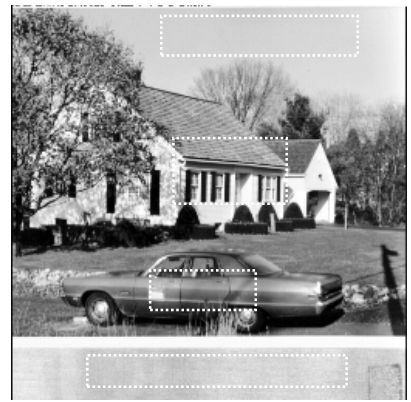

(i)

Figure 3 (a) Test image Car enhanced using: (b) HE; (c) BHEPLD; (d) BPPLHE; (e) MHE; (f) ESIHE; (g) AHEA; (h) ACEDP; and (i) the proposed AFCEDP technique. 


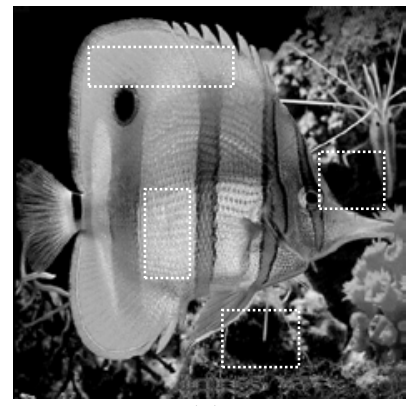

(a)

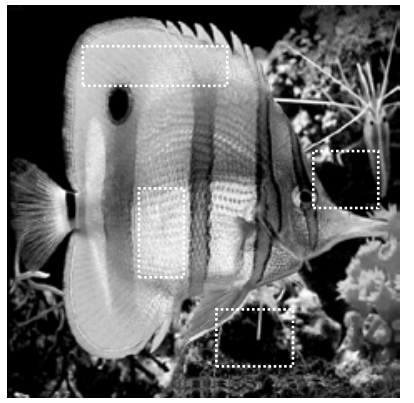

(d)

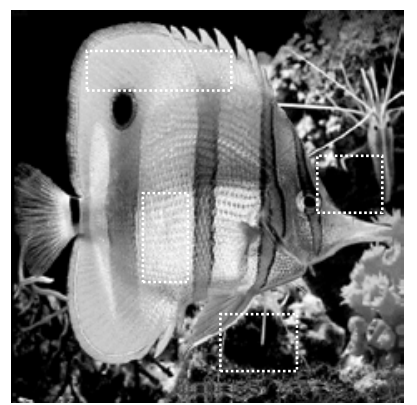

(g)

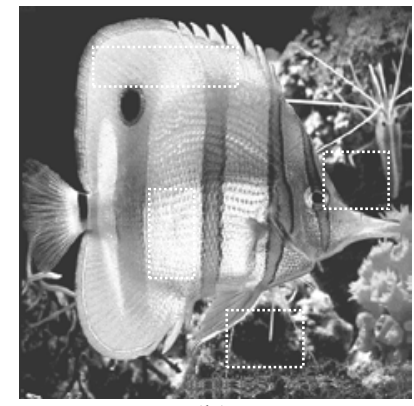

(b)

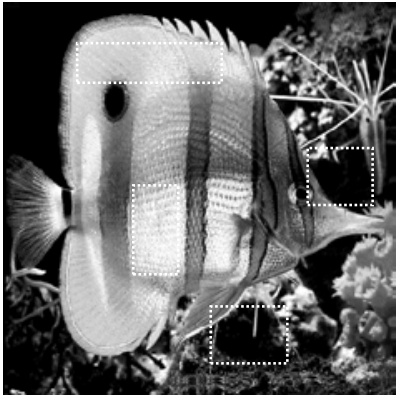

(e)

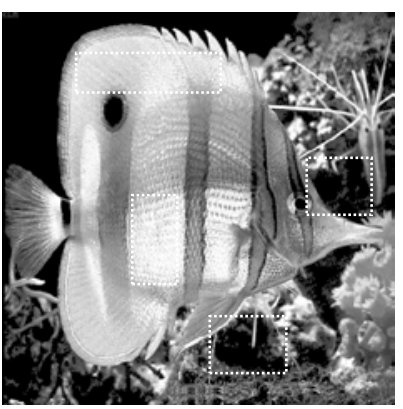

(h)

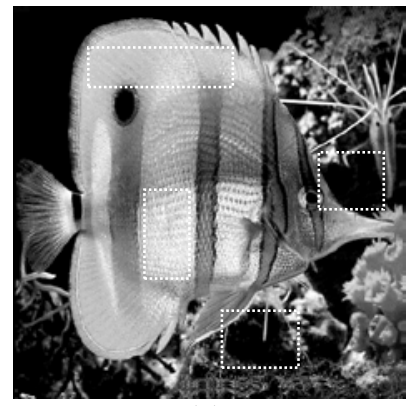

(c)

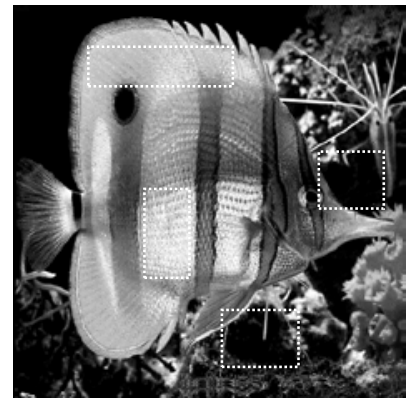

(f)

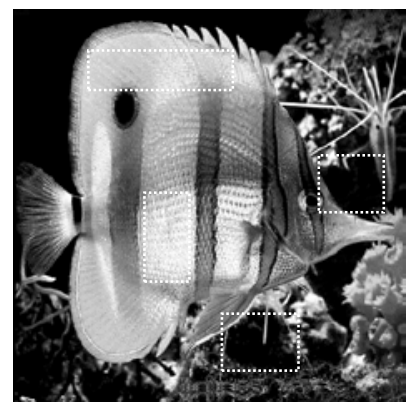

(i)

Figure 4 (a) Test image Fish enhanced using: (b) HE; (c) BHEPLD; (d) BPPLHE; (e) MHE; (f) ESIHE; (g) AHEA; (h) ACEDP; and (i) the proposed AFCEDP technique.

Table 1 suggests that the proposed AFCEDP technique performed the best among the techniques in terms of contrast enhancement. It has the highest output-input standard deviation and contrast improvement measurement. With the second ranking in the entropy measurement it was capable at retaining the image details during the enhancement process. 
Table 1 Average quantitative analysis results for 90 test images.

\begin{tabular}{ccccc}
\hline Techniques & Year & Entropy & Stand Dev. & Contrast (dB) \\
\hline HE & - & 5.805 & 2.700 & 67.512 \\
BHEPLD & 2010 & 6.374 & 2.474 & 64.454 \\
BPPLHE & 2010 & 6.496 & 2.263 & 64.974 \\
MHE & 2012 & 6.849 & 2.592 & 67.245 \\
ESIHE & 2013 & 6.865 & 2.286 & 66.735 \\
AHEA & 2012 & $\mathbf{6 . 9 3 4}$ & 2.048 & 66.932 \\
ACEDP & 2014 & 6.876 & 2.739 & 67.516 \\
AFCEDP (proposed) & - & 6.882 & $\mathbf{2 . 8 7 0}$ & $\mathbf{6 7 . 5 9 4}$ \\
\hline
\end{tabular}

It is worth mentioning that the AHEA technique was specifically designed to preserve the image entropy. The proposed AFCEDP technique was better than the conventional ACEDP technique in both detail preservation and contrast enhancement. Thus, this indicates that the integration of a fuzzy element aids yielded better enhancement results.

\subsection{Special Case: Cervical Cell Images and HEp-2 Cell Images}

With the encouraging results from the standard test images, the robustness of the proposed technique in enhancing microscopic medical images was further investigated. Here, cervical cell images and HEp-2 cell images were used as the test images. A total of 80 cervical cell images and 8 HEp- 2 cell images collected from Hospital Universiti Sains Malaysia, Kelantan, Malaysia, were enhanced using the proposed AFCEDP technique. Three out of these medical cell images are shown in Figure 5. The first row of images in Figure 5 shows the input images and the second row shows the images enhanced with the proposed AFCEDP technique.

It is obvious that both the original HEp-2 images and the cervical cell images have a relatively low contrast. The edges of the nucleus and the cytoplasm are unclear. The proposed AFCEDP technique successfully enhanced the contrast of the medical cell images while retaining most of the image details. In fact, when compared to the input images, the edges of both the nucleus and cytoplasm are more distinguishable.

The input and output entropy values are very similar, which confirms the AFCEDP technique's detail preservation capability. In addition, the AFCEDP technique shows an average improvement of $28.8 \%$ in terms of contrast improvement. 
Both the qualitative analysis and the quantitative analysis showed that the proposed AFCEDP technique effectively enhanced the standard images and the medical microscopic images.

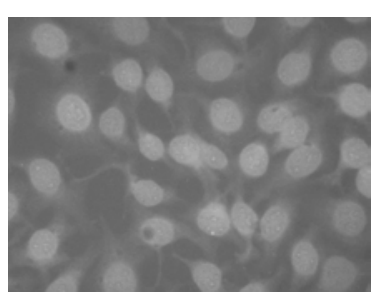

(a) $E=5.839$; $C=24.971$

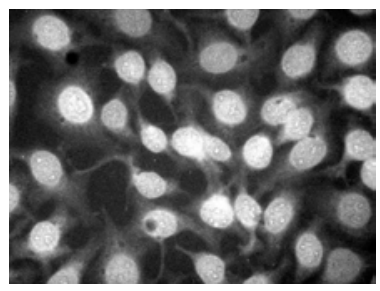

(d) $E=5.828$;

$C=35.958$;

$S D=9.107$

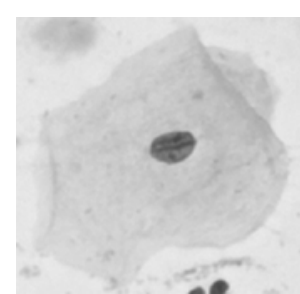

(b) $E=5.690$; $C=25.910$

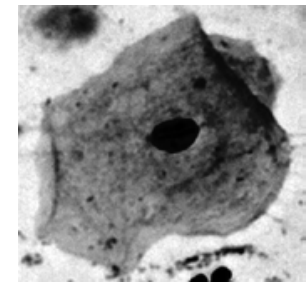

(e) $E=5.623$;

$C=36.847$;

$S D=7.357$

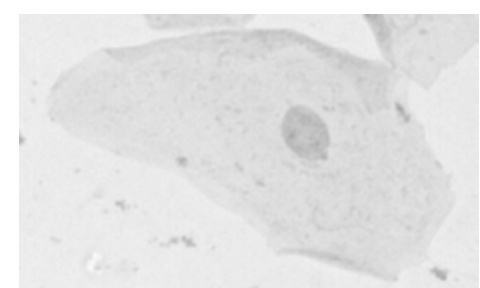

(c) $E=4.877$; $C=19.839$

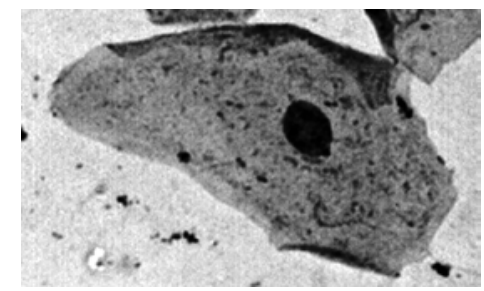

(f) $E=4.863$;

$C=36.090$;

$S D=10.113$

Figure 5 First row: (a) HEp-2_cell; (b) Cervical_cell_1; (c) Cervical_cell_2; Second row: (d) Enhanced_HEp-2_cell; (e) Enhanced_cervical_cell_1; and (f) Enhanced_cervical_cell_2.

\section{Conclusion}

This paper proposes a novel modification of the conventional Adaptive Contrast Enhancement Algorithm with Details Preserving (ACEDP) technique. The proposed Adaptive Fuzzy Contrast Enhancement with Details Preserving (AFCEDP) technique integrates the use of a fuzzy element in the categorization of the image and thus enables better selection of the clipping limits. The advantage of the proposed technique was proven by simulation results in which the AFCEDP technique outperformed the conventional ACEDP technique in terms of detail preservation and contrast enhancement. In future work, the effect of different membership functions, such as a Gaussian function, will be studied as an alternative to the trapezoidal-shaped membership functions implemented in the AFCEDP technique. 


\section{Acknowledgements}

The authors express their sincere thanks to the anonymous reviewers for their significant contributions to the improvement of the final paper. This study was partially supported by Universiti Sains Malaysia (USM) Research University Individual (RUI) grant entitled "Development of an Intelligent Auto-Immune Disease Diagnostic System by Classification of Hep-2 Immunofluorescence Patterns”, by National Cancer Council Malaysia (MAKNA), Malaysia, under project entitled "Development of an Intelligent Screening System for Cervical Cancer" and by Ministry of Higher Education (MOHE), Malaysia under MyPhD Scholarship.

\section{Reference}

[1] Gonzalez, R.C. \& Woods, R.E., Digital Image Processing, $2^{\text {nd }}$ ed., New Jersey: Prentice Hall, 2002.

[2] Yu, W., Qian, C. \& Baeomin, Z., Image Enhancement based on Equal Area Dualistic Sub-image Histogram Equalization Method, IEEE Transactions on Consumer Electronics, 45(1), pp. 68-75, 1999.

[3] Zhu, Y. \& Huang, C., Histogram Equalization Algorithm for Variable Gray Level Mapping, $8^{\text {th }}$ World Congress on Intelligent Control and Automation (WCICA), pp. 6022-6025, 2010.

[4] Abdullah-Al-Wadud, M., Kabir, M.H., Dewan, M.A.A. \& Oksam, C., A Dynamic Histogram Equalization for Image Contrast Enhancement, IEEE Transactions on Consumer Electronics, 53(2), pp. 593-600, 2007.

[5] Tang, J.R. \& Mat Isa, N.A., Adaptive Contrast Enhancement Algorithm with Details Preserving, Proceedings of 2014 International Conference on Electrical Engineering Computer Science and Informatics, Institute of Advanced Engineering and Science IAES, 2014.

[6] Kim, Y.-T., Contrast Enhancement using Brightness Preserving Bihistogram Equalization, IEEE Transactions on Consumer Electronics, 43(1), pp. 1-8, 1997.

[7] Ooi, C.H., Sia, N.P.K. \& Ibrahim, H., Bi-histogram Equalization with a Plateau Limit for Digital Image Enhancement, IEEE Transactions on Consumer Electronics, 55(4), pp. 2072-2080, 2009.

[8] Der, C.S. \& Ramli, A.R., Minimum Mean Brightness Brror Bi-histogram Equalization in Contrast Enhancement, IEEE Transactions on Consumer Electronics, 49(4), pp. 1310-1319, 2003.

[9] Der, C.S. \& Ramli, A.R., Contrast Enhancement using Recursive Meanseparate Histogram Equalization for Scalable Brightness Preservation, IEEE Transactions on Consumer Electronics, 49(4), pp. 1301-1309, 2003. 
[10] Sim, K.S., Tso, C.P. \& Tan, Y.Y., Recursive Sub-image Histogram Equalization applied to Gray Scale Images, Pattern Recognition Letters, 28(10), pp. 1209-1221, 2007.

[11] Sheet, D., Garud, H., Suveer, A., Mahadevappa, M. \& Chatterjee, J., Brightness Preserving Dynamic Fuzzy Histogram Equalization, IEEE Transactions on Consumer Electronics, 56(4), pp. 2475-2480, 2010.

[12] Abdullah-Al-Wadud, M., A Modified Histogram Equalization for Contrast Enhancement Preserving The Small Parts in Images, International Journal of Computer Science and Network Security, 12, 2012.

[13] Zhu, Y. \& Huang, C., An Adaptive Histogram Equalization Algorithm on the Image Gray Level Mapping, Physics Procedia, 25(0), pp. 601-608, 2012.

[14] Ooi, C.H. \& Mat Isa, N.A., Adaptive Contrast Enhancement Methods with Brightness Preserving, IEEE Transactions on Consumer Electronics, 56(4), pp. 2543-2551, 2010.

[15] Ooi, C.H., New Histogram Equalization Based Detail and Brightness Preserving Techniques for Digital Images, Master of Science, School of Electrical and Electronic Engineering, Universiti Sains Malysia, 2010.

[16] Singh, K. \& Kapoor, R., Image Enhancement Using Exposure Based Sub Image Histogram Equalization, Pattern Recognition Letters, 36, pp. 1014, 2014.

[17] Shannon, C.E., A Mathematical Theory of Communication, Bell Systems Technical Journal, 27(3), pp. 379-423, 1948.

[18] Zadbuke, A.S., Brightness Preserving Image Enhancement Using Modified Dualistic Sub Image Histogram Equalization, International Journal of Scientific \& Engineering Research, 3(2), 2012.

[19] Khan, M.F., Khan, E. \& Abbasi, Z.A., Weighted Average Multi Segment Histogram Equalization for Brightness Preserving Contrast Enhancement, In: IEEE International Conference on Signal Processing, Computing and Control (ISPCC), pp. 1-6, 2012.

[20] Liang, K., Ma, Y., Xie, Y., Zhou, B. \& Wang, R., A New Adaptive Contrast Enhancement Algorithm for Infrared Images Based on Double Plateaus Histogram Equalization, Infrared Physics \& Technology, 55(4), pp. 309-315, 2012.

[21] Chang-Jiang, Z., Meng-Yin, F., Jin, M. \& Qi-Hong, Z., Approach to Enhance Contrast of Infrared Image Based on Wavelet Transform, Journal of Infrared and Millimeter Waves, 23(2), pp. 119-124, 2004.

[22] CVG-UGR-Database, http://decsai.ugr.es/cvg/dbimagenes, June $1^{\text {st }}$, 2014. 